\title{
Alternative polyadenylation signals and promoters act in concert to control tissue-specific expression of the Opitz Syndrome gene MIDI Jennifer Winter*1, Melanie Kunath ${ }^{1}$, Stefan Roepcke ${ }^{1,3}$, Sven Krause ${ }^{1}$, Rainer Schneider ${ }^{2}$ and Susann Schweiger ${ }^{1,4,5}$
}

\author{
Address: ${ }^{1}$ Max-Planck Institute for Molecular Genetics, Berlin-Dahlem, Germany, ${ }^{2}$ Institute of Biochemistry, University Innsbruck, Austria, \\ ${ }^{3}$ ALTANA Pharma AG, Preclinical Research Bioinformatics, Konstanz, Germany, ${ }^{4}$ Department of Dermatology, Charité-Hospital, Berlin, Germany \\ and ${ }^{5}$ Department of Neuroscience and Pathology, College of Medicine, University of Dundee, Dundee, UK \\ Email: Jennifer Winter* - winter@molgen.mpg.de; Melanie Kunath - kunath@molgen.mpg.de; \\ Stefan Roepcke - stefan.roepcke@altanapharma.com; Sven Krause - krause_s@molgen.mpg.de; Rainer Schneider - rainer.schneider@uibk.ac.at; \\ Susann Schweiger - schweige@molgen.mpg.de \\ * Corresponding author
}

Published: 15 November 2007

BMC Molecular Biology 2007, 8:105 doi:10.1 186/147|-2199-8-105

This article is available from: http://www.biomedcentral.com/I47/-2/99/8/105

(C) 2007 Winter et al; licensee BioMed Central Ltd.

This is an Open Access article distributed under the terms of the Creative Commons Attribution License (http://creativecommons.org/licenses/by/2.0), which permits unrestricted use, distribution, and reproduction in any medium, provided the original work is properly cited.
Received: 16 April 2007

Accepted: 15 November 2007

\begin{abstract}
Background: Mutations in the X-linked MID / gene are responsible for Opitz G/BBB syndrome, a malformation disorder of developing midline structures. Previous Northern blot analyses revealed the existence of at least three MIDI transcripts of differing lengths.

Results: Here we show that alternative polyadenylation generates the size differences observed in the Northern blot analyses. Analysis of EST data together with additional Northern blot analyses proved tissue-specific usage of the alternative polyadenylation sites. Bioinformatic characterization of the different 3'UTRs of MIDI revealed numerous RNA-protein interaction motifs, several of which turned out to be conserved between different species. Furthermore, our data suggest that mRNA termination at different polyadenylation sites is predetermined by the choice of alternative 5'UTRs and promoters of the MID I gene, a mechanism that efficiently allows synergistic function of $5^{\prime}$ and 3 'UTRs.

Conclusion: MIDI expression is tightly regulated through concerted action of alternative promoters and alternative polyadenylation signals both during embryonic development and in the adult.
\end{abstract}

\section{Background}

Mutations in the X-linked MID1 gene cause Opitz G/BBB syndrome (OS). OS is a congenital malformation syndrome characterized by defective ventral midline development with the main features being ocular hypertelorism and hypospadias. Additional abnormalities such as cleft lip and palate, laryngo-tracheal fistulas, heart defects, imperforate anus and mental retardation may also be present.
Recently we found that the MID1 protein associates with microtubules [1] and triggers ubiquitination and degradation of the microtubule-associated protein phosphatase 2a (PP2A) upon interaction with the $\alpha 4$ protein [2]. MID1 loss-of-function mutations, as seen in OS patients, thus cause accumulation of microtubule-associated PP2A and hypophosphorylation of its target proteins. 
The MID1 mRNA is subject to extensive alternative splicing [3]. Also, several 5'-untranslated regions have been identified and the use of five alternative promoters results in the production of additional MID1 transcript isoforms [4].

The expression pattern of MID1 has been investigated by Northern blot analyses and in situ hybridization [5-8]. In humans, three transcripts of $\sim 7 \mathrm{~kb}, \sim 4.5 \mathrm{~kb}$ and $\sim 3.5 \mathrm{~kb}$ were observed in all fetal and adult tissues analyzed [6,9]. Remarkably, the coding sequence of MID1 accounts for only $\sim 2 \mathrm{~kb}$, and the size differences between the known MID1 sequence and the transcripts cannot be explained by alternative splicing of either the coding region or 5 'UTR. However, splicing and/or alternative polyadenylation of the 3'UTR have not been investigated so far.

The 3'UTRs of many genes have been shown to be involved in pleiotropic regulatory functions, such as RNA localization, mRNA degradation and stabilization, and translational control. In the present work we describe the identification of several alternative polyadenylation sites in the human MID1 3'UTRs which give rise to transcripts with four different 3'UTRs and tissue-specific expression patterns.

To identify putative regulatory structures we have characterized the MID1 3'UTR with bioinformatic tools and report the presence of putative target sites for RNA binding proteins. Notably, we identified several AU-rich elements (AREs) and cytoplasmic polyadenylation elements (CPEs). As proteins binding to both AREs and CPEs are known to be key regulators of mRNA stability and/or translation, our results suggest a tight control of MID1 expression through the different 3'UTRs. Intriguingly, we also found that specific polyadenylation signals are arrayed with distinct 5'UTRs and promoters of the MID1 gene, indicating that polyadenylation is a promoterdriven process.

\section{Results \\ EST data indicate alternative polyadenylation of the MIDI gene}

Previous Northern blot analyses of human PolyA ${ }^{+}$RNA showed MID1 transcripts of $\sim 7 \mathrm{~kb}, \sim 4.5 \mathrm{~kb}$ and $\sim 3.5 \mathrm{~kb}$ $[6,9]$. As these size differences cannot be explained by alternative splicing of the coding sequence or the 5'UTR, we hypothesized the existence of alternative polyadenylation sites (poly(A) sites) in the 3'UTR. To test this hypothesis we analyzed human EST data overlapping the MID1 3'UTR. A review of the human EST database indicated at least three alternative poly(A) sites (Fig. 1a), which we named ESTa, $b$ and c. Whereas ESTa and c contain consensus polyadenylation signals at their 3 'ends and therefore seem to terminate at real polyadenylation sites, ESTb does not contain such a signal. A stretch of oligo-A present at the 3'end of ESTb pointed to putative mis-priming of polyT-primers as a likely cause of this artifactual polyadenylation site (Fig. 1a). While 53 ESTs overlap ESTc, only 23 ESTs correspond to ESTa (see additional file 1); this likely reflects preferential use of the polyadenylation site corresponding to ESTc.

\section{Alternative polyadenylation of the MIDI gene in different species}

To confirm alternative polyadenylation experimentally we performed 3'RACE with cDNA derived from human fibroblasts (Fig 1b). Fibroblasts were chosen for this analysis because they express MID1 at a moderate level. Interestingly, sequencing of the PCR products revealed four different polyadenylation sites which we named PAS1-4 (Fig. 1a and 1b). Two of them match the EST data: PAS1 corresponded to ESTa and PAS3 to ESTc; however, no EST data were available for PAS2 and PAS4 (Fig. 1a).

Polyadenylation signals consisting of an upstream element (AAUAAA) and a downstream U-rich or GU-rich element are in close proximity to all four poly(A) sites (PAS1-PAS4, see additional file 2), and sequence comparison showed that all four upstream elements are conserved between human and dog. In contrast only some of the human upstream elements are conserved in other species. Whereas the element upstream of PAS2 is conserved between human, opossum and chicken, the element upstream of PAS3 is conserved between human and rat (see additional file 3 ). To test experimentally whether the MID1 mRNA is alternatively polyadenylated in other species we performed 3'RACE on cDNA from rat brain, a tissue known to express high levels of MID1 (Fig. 1b). Sequencing of the PCR products revealed three alternative polyadenylation sites, rPAS1-3 (Fig. 1b) with rPAS3 corresponding to PAS3 of the human MID1 gene, and rPAS1 and rPAS2 probably representing species-specific sites.

\section{3'UTR 4 directs expression of tissue specific transcripts}

To test for expression of the human transcript terminated by PAS4, we hybridized a specific riboprobe (nbPAS4; Fig. 1b) against commercially available Northern blots containing human polyA ${ }^{+}$RNA extracted from a variety of fetal and adult tissues. In contrast to the picture obtained with a probe detecting the MID1 open reading frame, which showed ubiquitous expression of a $\sim 7 \mathrm{~kb}$ transcript (Quaderi et al. 1997), a transcript of similar size could only be observed in fetal liver and skeletal muscle with nbPAS4 (Fig. 2a, arrows). Additionally, a variety of shorter and longer transcripts were detected in heart, skeletal muscle, liver and fetal liver. Among them a $\sim 2 \mathrm{~kb}$ transcript was identified in both adult and fetal liver, making it solely a liver-specific transcript (Fig. 2a, arrow). To further characterize these transcripts we performed 5'RACE 


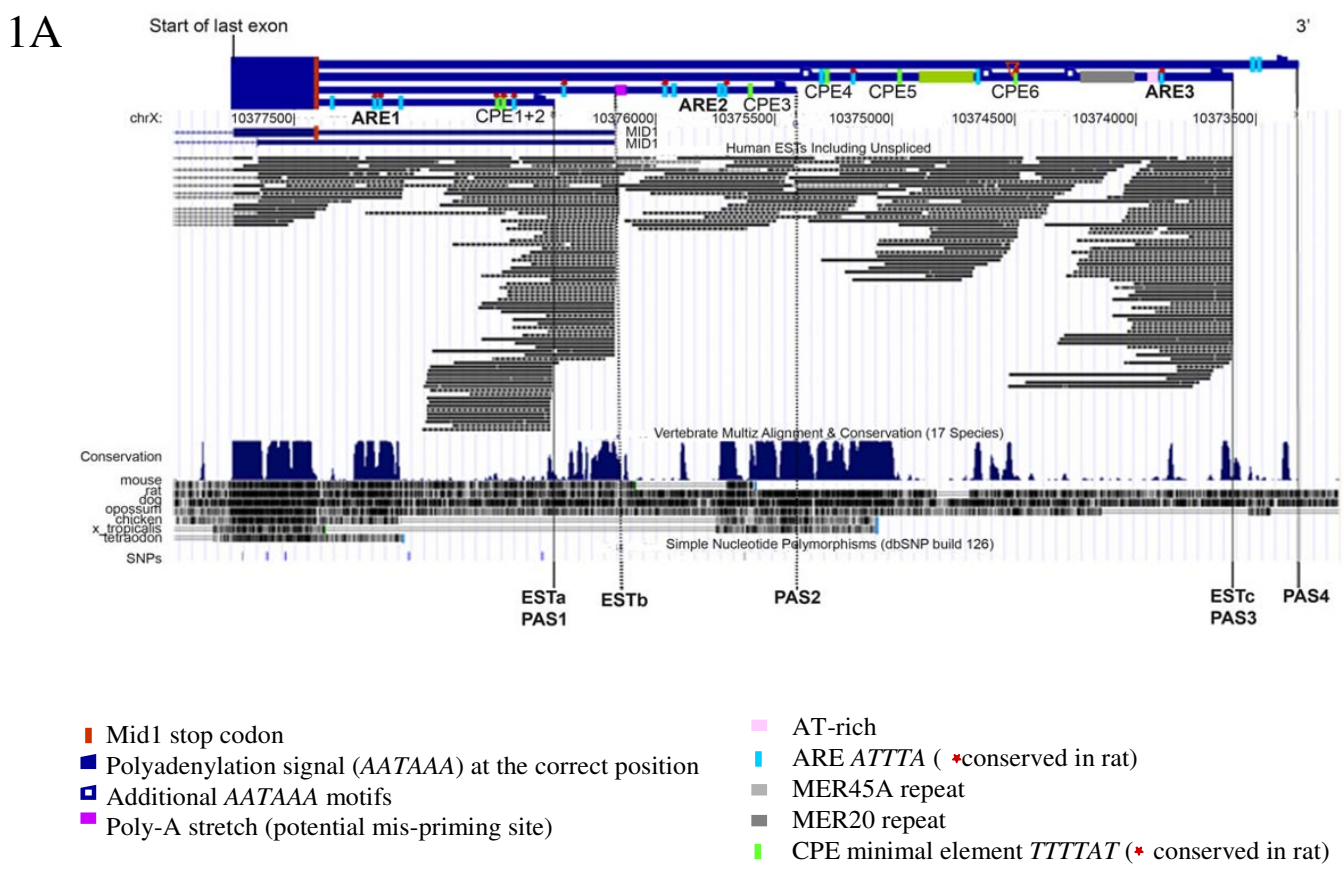

\section{$1 \mathrm{~B}$}
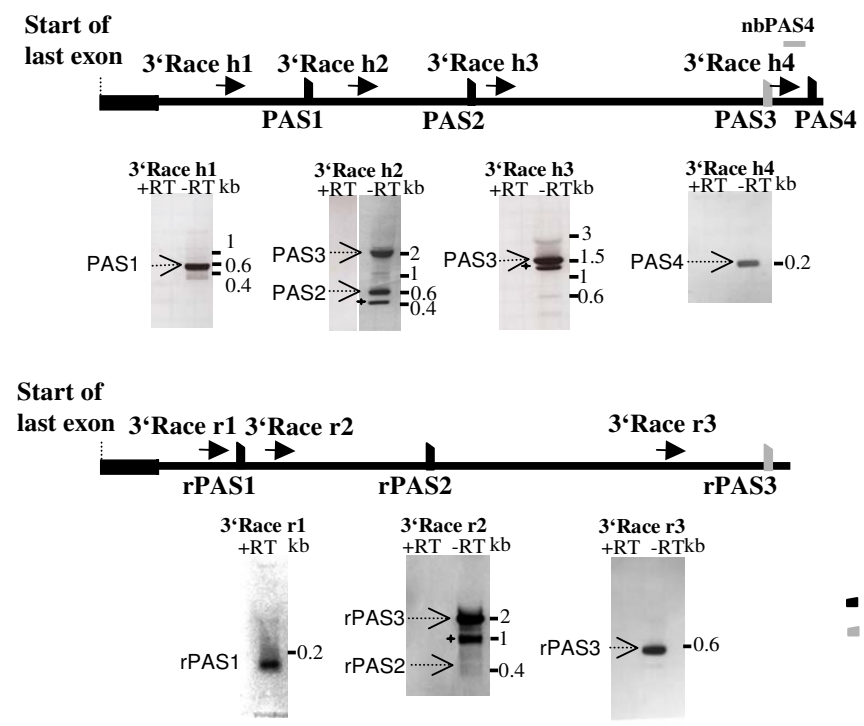

- polyadenylation signal

- conserved polyadenylation signal

\section{Figure I}

Alternative polyadenylation sites in the MIDI mRNAs of human (PASI-PAS4) and rat (rPASI-rPAS3). (A) The 3'UTR of human MIDI containing alternative polyadenylation sites identified in this study together with data on mRNAs, ESTs and conservation obtained from the UCSC genome browser March 2006 assembly. Regulatory motifs are highlighted in different colors. (B) Ethidium bromide gels of 3'RACE experiments. Locations of primers are indicated in the cartoons (arrows). Asterisks indicate unspecific products. PAS3 and rPAS3 are homologues. The nucleotide sequences of novel 3'ends have been submitted to Genbank with accession numbers EF217423, EF217424, EF217425, EF217426, EF217427, EF217428, and EF217429. The position of the northern probe nbPAS4 is indicated. 
$2 \mathrm{a}$

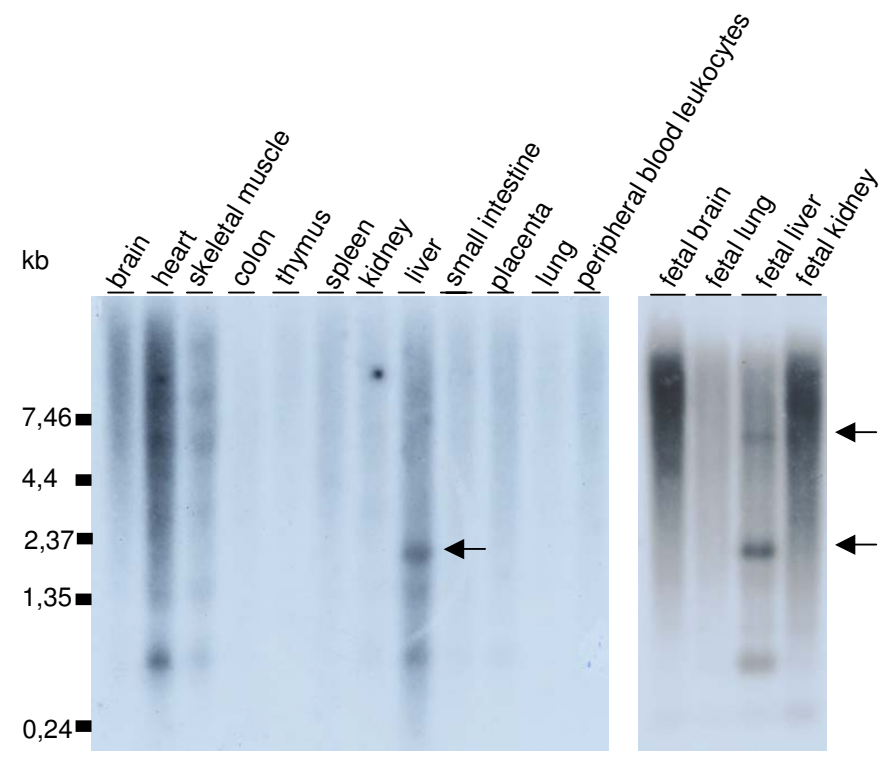

$\mathrm{b}$

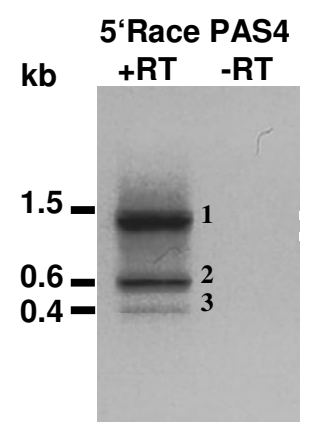

polyadenylation signal •

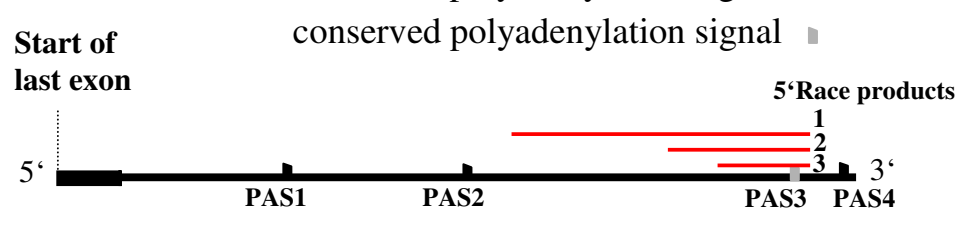

\section{Figure 2}

Northern blot and 5'RACE analysis of human 3'UTR4. (A) Fetal and adult multiple-tissue Northern blots hybridized with a riboprobe detecting a region between PAS3 and PAS4 of the human 3'UTR. Arrows indicate MIDI transcripts. (B) 5 'RACE products obtained with primers located downstream of PAS3. The nucleotide sequences of 5'RACE products have been submitted to Genbank with accession numbers EF532594, EF532595, EF532596.

experiments on cDNA derived from human fetal liver with a gene-specific primer located downstream of PAS3 (Fig. 2b). Interestingly, sequencing of the PCR products revealed three unspliced transcripts of different lengths with transcription start sites located in the 3'UTR region upstream of PAS3 (Fig. 2b).

\section{MIDI transcripts starting from alternative 5'UTRs end at specific polyadenylation sites}

Previous Northern blot analyses showed MID1 transcripts of $\sim 7 \mathrm{~kb}, \sim 4.5 \mathrm{~b}$ and $\sim 3.5 \mathrm{~kb}[6,9]$, thus indicating that all alternative polyadenylation signals are connected to the full-length coding sequence. However, as human MID1 has five alternative promoters and 5'UTRs [4], we tested for preferential and regulated choice of polyadenylation signals in transcripts starting from alternative promoters. RT-PCR experiments were performed using RNA derived from human fibroblasts with primers connecting alternative 5'UTR exons $1 \mathrm{a}, 1 \mathrm{c}$ and $1 \mathrm{e}$ to regions upstream of PAS1 (primer set 1), PAS2 (primer set 2) and PAS3 (primer set 3) (Fig. 3a and 3b). Of note, primer set 1 amplified transcripts with poly(A) tails at PAS1-4 whereas primer set 2 amplified transcripts with poly(A) tails at PAS2-4 and primer set 3 exclusively amplified transcripts with poly(A) tails at PAS3-4. For sequencing, PCR products were excised from the gel and cloned into the pGEM-T Easy vector.

Concerning exon 1c, products were amplified with each of the different 3'UTR primer sets (Fig. 3b). In view of the tis- 

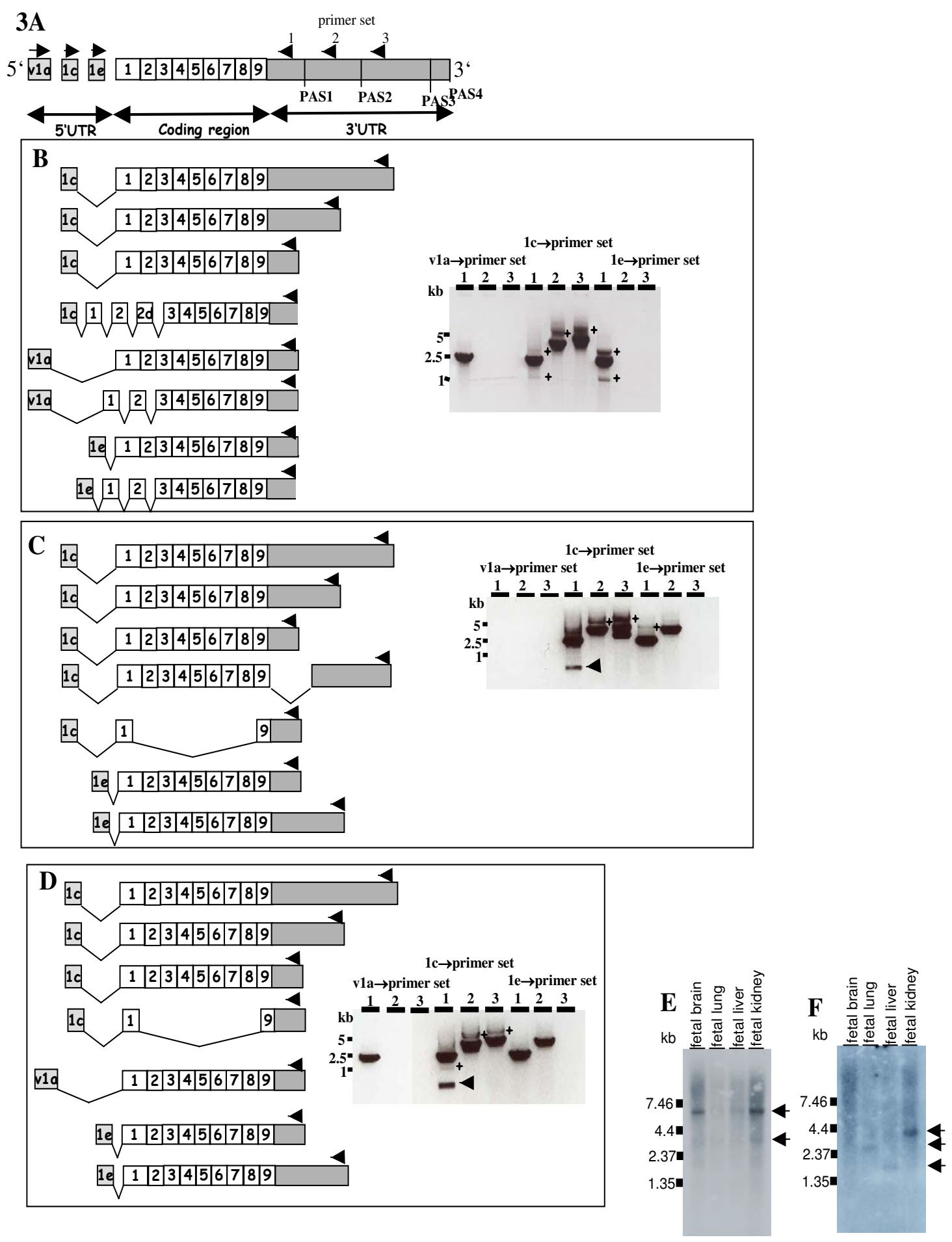

Figure 3

Identification of full-length MIDI transcripts. (A) 5'UTR exons, coding region and 3'UTR of MID I. Arrows indicate location of primers. (B-D) Ethidium bromide stained gels of RT-PCR products obtained using RNA from fibroblasts (B), testis (C) or fetal brain (D). Asterisks indicate products that could not be sequenced due to their low abundance. RT-PCR products obtained with combinations of primer sets I-3, with primers located in 5'UTR exons Ic, vla or I e. (E, F) Fetal tissue Northern blots hybridized with probes corresponding to 5'UTR exons Ic (E) or le (F). Arrows indicate MIDI transcripts. 
sue-restricted expression of PAS4, this result clearly shows that transcripts starting in exon $1 \mathrm{c}$ are polyadenylated at PAS3 and thus correspond to the $7 \mathrm{~kb}$ transcript seen on Northern blots. However, it remains unclear whether there actually are transcripts that start in exon $1 \mathrm{c}$ and terminate at PAS1 or PAS2. Sequencing of the cloned PCR products revealed the constitutive MID1 coding sequence to be present in five out of six clones (Fig. 3b). One clone represented an in-frame splice variant containing the short variant of exon 1, alternatively spliced exon $2 \mathrm{~d}$ and constitutive exons 2-9 [3] (Fig. 3b). Because of the comparable sizes of the two transcripts, the RT-PCR products of the alternative splice variant and the constitutive transcript could not be separated on the agarose gel.

RT-PCR with primers located in Exv1a amplified products only in combination with reverse primers located upstream of PAS1, indicating preferential polyadenylation at PAS1 in transcripts derived from promoter 1a (Fig. $3 \mathrm{~b})$. However, as the primers located upstream of PAS2 showed inconsistent results in other experiments (see below), we cannot exclude the use of PAS2 with Exv1a. Again, sequencing of three clones confirmed the specificity of the RT-PCR reaction and revealed the presence of the constitutive coding sequence in two of them and the presence of an alternative splice variant containing the short variant of exon 1 in the third (Fig. 3b).

The use of forward primers located in exon 1e led to inconsistent results. In two out of three independent experiments we obtained products when using reverse primers located upstream of PAS3. While we were not able to clone these products, the transcript sizes indicated the presence of the entire 3'UTR sequence in those transcripts. However, when we used reverse primers located upstream of PAS2, no products were obtained in any of the experiments. RT-PCR with reverse primers located upstream of PAS1 amplified transcripts in every experiment (Fig. 3b). Characterization of these transcripts revealed the presence of the constitutive coding sequence in five out of six sequenced clones and a splice variant containing the short variant of exon 1 in the sixth (Fig. 3b).

Our RT-PCR experiments indicate preferential and regulated choice of polyadenylation signals for transcripts starting from each single MID1 promoter. To test whether this phenomenon is a characteristic of fibroblasts or a general regulatory mechanism of MID1 expression we performed RT-PCR experiments using RNA derived from two additional human tissues, namely testis and fetal brain (Fig. 3c and 3d). Again, we obtained products with each of the different 3'UTR primer sets when forward primers were located in exon 1c (Fig. 3c and 3d) and only obtained products from fetal brain with primer set 1 when forward primers were located in Exv1a (Fig. 3d). When we used RNA from testis we couldn't obtain any products with primers located in Exv1a indicating that Exv1a is not expressed in this tissue. With exon 1e primers products were amplified only with primer sets 1 and 2 indicating termination at PAS2 in these tissues.

Sequencing of the two cloned products revealed the constitutive MID1 coding sequence when primers where located in Exv1a or exon 1e. Concerning exon 1c we additionally obtained two alternative splice variants (Fig. 3c and $3 \mathrm{~d}$ ). One short variant, which was present in both tissues, testis and fetal brain (Fig. 3c and 3d, arrows), contained constitutive exons 1 and 9 . The second splice variant lacked part of the 3'UTR but contained the whole constitutive coding region.

In confirmation of the RT-PCR experiments, probes specifically detecting exon $1 c$ or exon $1 e$ were hybridized against commercially available Northern blots containing polyA $^{+}$RNA extracted from a variety of human fetal tissues (Fig. $3 e$ and $3 \mathrm{f}$ ). A $\sim 7 \mathrm{~kb}$ and a weaker $\sim 3.5 \mathrm{~kb}$ transcript were detected in all fetal tissues analyzed using a probe hybridizing to exon 1c (Fig. 3e) confirming termination of exon 1c transcripts at PAS3 (predominantly) and PAS1. In contrast, a probe hybridizing to exon 1e detected a $~ 3.5$ kb transcript but not a $\sim 7 \mathrm{~kb}$ transcript (Fig. 3f). This hybridization pattern indicated that the $7 \mathrm{~kb}$ transcript, which would use the PAS3 polyadenylation signal, is a rare mRNA when transcription is initiated by use of promoter e. However, while expression of the $\sim 3.5 \mathrm{~kb}$ transcript was high in fetal kidney, weak expression of the same transcript could be detected in the other fetal tissues by autoradiography prior to the final washing of the membrane (data not shown). Also we observed expression of smaller transcripts of $\sim 2.5 \mathrm{~kb}$ in fetal lung and $\sim 1.5$ $\mathrm{kb}$ in fetal liver, suggesting that promoter e drives expression of smaller splice variants in addition to the expression of the constitutive MID1 coding sequence (Fig. 3f). However, because hybridization was carried out with a double-stranded DNA-probe, these smaller transcripts might also be overlapping antisense transcripts. Remarkably, in contrast to our RT-PCR experiments we could not detect any transcripts in fetal brain, which indicates their low expression. By direct comparison of the two Northern blots (Fig. $3 e$ and $3 \mathrm{f}$ ) the two main transcripts of each promoter variant $(7 \mathrm{~kb}$ when exon $1 \mathrm{c}$ is used and $3.5 \mathrm{~kb}$ when exon $1 \mathrm{e}$ is used) both appear to be highly expressed in fetal kidney while expression levels of these transcripts and those of smaller sizes appear to vary in all other tissues.

\section{The MIDI 3'UTR contains highly conserved regulatory motifs, which are bound by interacting proteins}

In order to screen for functionally relevant sequences within the 3'UTR of MID1, evolutionary conservation 
between human, rat and dog MID1 3'UTRs was analyzed. While the overall sequence identity (from the end of the coding sequence through to PAS3 in the rat and through to PAS4 in the dog) is $76 \%$ between human and rat and $80 \%$ between human and dog, some blocks of stronger sequence similarity are present - the strongest starting $1,852 \mathrm{bp} \mathrm{3'}$ of the translational stop codon, spanning 503 bp, and having a sequence similarity of $88 \%$ between human and rat. Parts of the 3'UTR are conserved even between human/rat and more distantly related species like Xenopus tropicalis and Tetraodon (Fig. 1a), indicating that the MID1 3'UTR is under strong selective pressure.

Conservation of the sequence of the MID1 3'UTR suggests the presence of regulatory motifs, such as for the binding of proteins. Bioinformatic analysis indeed identified several putative protein-binding motifs. In addition to motifs like cytoplasmic polyadenylation elements with the consensus sequence TTTTAT [10] and additional polyade- nylation signals, we found AU-rich elements (AREs) with the sequence ATTTA in all parts of the 3'UTR (Fig. 1a, 4a and additional file 4). Some of these short ARE motifs were found to be parts of much longer AU-rich sequences which may indicate their functional relevance [11] (Fig. 4a). AREs have been shown to influence RNA stability and/or to control translation of a number of genes [1113].

Particularly ARE1 seemed to be of potential functional relevance because it comprises a long AU-rich sequence, which is highly conserved in various species (Fig. 4a). To test for binding of interacting proteins to this motif a radioactively labelled transcript corresponding to ARE1 was incubated with HeLa cell lysate and subsequent UVcrosslinking was performed. Complexes were resolved by electrophoresis through SDS acrylamide gels and dried gels were exposed to X-ray film. Interestingly, this method identified several proteins of $\sim 78$ and $30-45 \mathrm{kD}$ that had

\section{$4 \mathrm{~A}$}

ARE 1 5
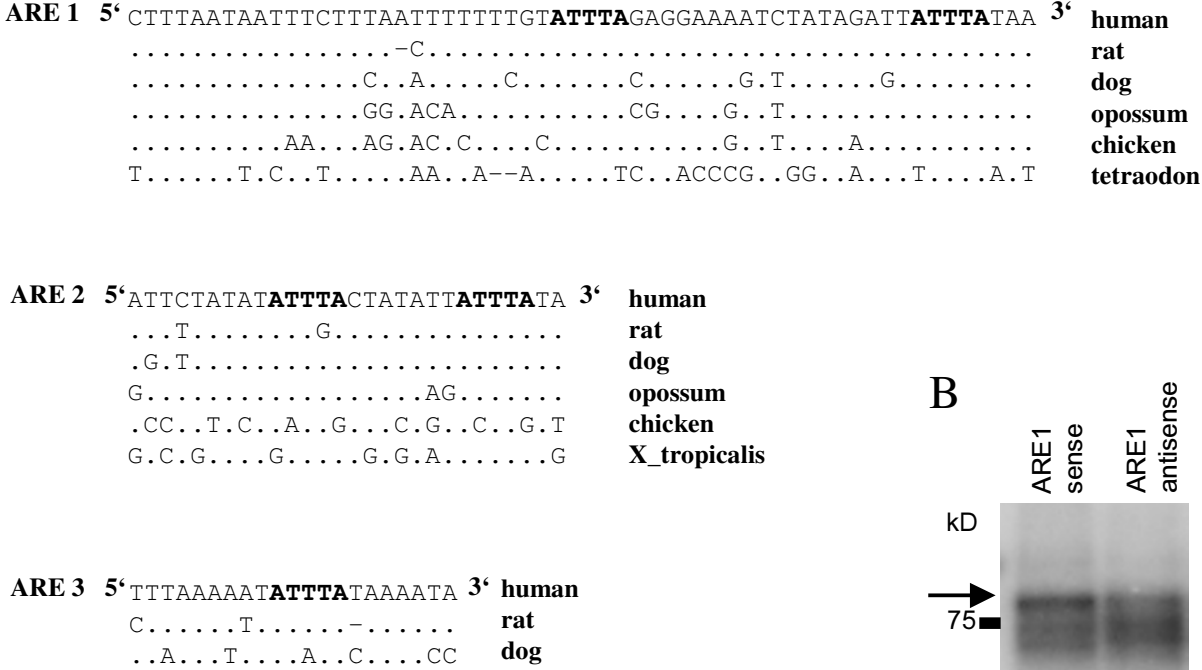

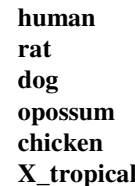

huma

chicken

X_tropicalis

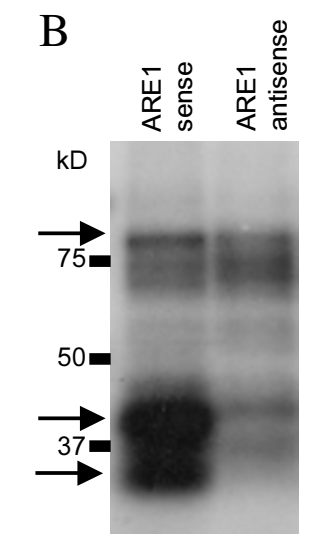

AUF1

HuR

\section{Figure 4}

Regulatory motifs in the 3'UTR of the human MIDI gene. (A) Sequences of ARE motifs I-3 with surrounding AU-rich sequences. (B) UV-crosslink with protein lysate from HeLa cells and the AREI motif. Arrows indicate proteins that bound the AREI sense transcript with much higher affinity than the antisense negative control. (C) Western blot analyses of RNA-protein pulldowns with protein lysate from HeLa cells and the AREI motif. HuR and AUFI are detected using specific antibodies. 
bound the sense ARE1 transcript but not the antisense control (Fig. 4b). In a next step we tried to identify proteins which interact with the ARE1 motif. As candidates we considered the ARE-binding proteins HuR and AUF1 because they have sizes between 32 and $45 \mathrm{kD}$ which correspond to those of the proteins seen in the UV-assay. To test for binding of the candidate proteins we performed a RNA-protein pulldown assay by incubating a biotin labelled transcript corresponding to ARE1 with HeLa cell lysate. RNA-protein complexes were subsequently pulled down with streptavidin magnetic beads. Complexes were resolved by electrophoresis through SDS acrylamide gels. Western blot analyses with specific antibodies directed against AUF1 and HuR showed a clear binding of both proteins to the ARE1 sense RNA, while only little AUF1 protein and almost no HuR had bound to the ARE1 antisense RNA (Fig. 4c).

\section{Discussion}

Alternative polyadenylation is a widespread mechanism of gene regulation in mammals and is often associated with specific tissue/cell types and/or developmental stages [14-17]. Previous Northern blot analyses of human fetal and adult tissues identified MID1 transcripts of $\sim 7 \mathrm{~kb}$, $\sim 4.5 \mathrm{~kb}$ and $\sim 3.5 \mathrm{~kb}[6,9]$. Here we show that tissue-specific alternative polyadenylation in the MID1 gene underlies the observed size differences. Interestingly, usage of the identified polyadenylation sites appears to be determined by the choice of alternative promoters, which themselves contribute to differential MID1 expression [4]. In a bioinformatic approach we further found numerous putative RNA-protein interaction motifs in the MID1 3'UTRs, several of which turned out to be conserved between human and other species.

We found that the human MID1 3'UTR contains four polyadenylation sites, PAS1-PAS4. Polyadenylation at PAS1 results in a $3.5 \mathrm{~kb}$ transcript and usage of PAS2 leads to a $4.5 \mathrm{~kb}$ transcript. Due to a size difference of only 250 bp, mRNAs polyadenylated at PAS3 and PAS4 appear as a single $\sim 7 \mathrm{~kb}$ band on Northern blots.

In order to differentiate between the transcripts using either PAS3 or PAS4 we hybridized a riboprobe exclusively detecting the fourth part of the 3'UTR against commercially available Northern blots. In contrast to ubiquitous expression of the $7 \mathrm{~kb}$ transcript detected with a probe corresponding to the MID1 open reading frame (Quaderi et al. 1997), we saw expression of the PAS4 $7 \mathrm{~kb}$ transcript to be restricted to skeletal muscle and fetal liver. Hence, these experiments prove tissue restriction of the PAS4 transcripts and ubiquitous expression of the PAS3 transcripts and indicate that PAS3 is the constitutive polyadenylation signal. Remarkably, besides the $\sim 7 \mathrm{~kb}$ transcript, shorter variants of $\sim 2 \mathrm{~kb}, \sim 1.35 \mathrm{~kb}$ and $\sim 900 \mathrm{bp}$ could be observed when using the PAS4 specific riboprobe. The use of a single stranded riboprobe for Northern blot analyses excluded the possibility that these transcripts are overlapping antisense transcripts. 5'RACE showed that these transcripts are unspliced and have transcription starts which are located in the 3'UTR. Several points indicate that these are full-length transcripts. First, the overall sizes of transcripts 1 and 2 approximately match the sizes of the $2 \mathrm{~kb}$ and $0.9 \mathrm{~kb}$ Northern bands detected in the lane loaded with RNA from fetal liver (Fig. $2 \mathrm{a}$ and $2 \mathrm{~b}$ ). An additional smaller $0.4 \mathrm{~kb}$ transcript amplified by 5'RACE did not show up on the Northern blot which might be due to its low expression (Fig. 2b). Secondly, all three transcripts contain a distinct sequence motif which is found exclusively in transcription start sites derived from 3'UTRs [15], namely a triple G at the -3 to 1 position. In addition to the triple G, Carninci et al. [15] mentioned a highly conserved region located 40 to 90 bases downstream of 3'UTR transcription start sites. Concerning the MID1 transcripts, conservation of the +40 to +90 region is not higher than that of the remaining 3 'UTR. As PAS4 is poorly conserved in other species this polyadenylation site might be human specific and therefore a high conservation of the +40 to +90 region might not be expected. Although the functions of transcripts with transcription starts in 3'UTRs are unclear it has been suggested that they might regulate downstream genes which are encoded on the opposite strand using a sense-antisense mechanism [15]. The next neighbouring gene, the CLCN4 gene, is located at a distance of $\sim 350 \mathrm{~kb}$ downstream of MID1. As this gene is encoded on the opposite strand compared to MID1 such a sense-antisense regulation seems possible. On the other hand the three identified transcripts might encode short proteins. However, inspection of the sequence of transcript 1 which also contains the sequences of transcripts 2 and 3 revealed the longest protein sequence to be 83 amino acids with no conserved domains.

Tian et al. estimated that $~ 54 \%$ of all human genes and $\sim 32 \%$ of all mouse genes use alternative polyadenylation sites [18]. Many human polyadenylation signals used are conserved in their rodent orthologs. Interestingly, concerning the MID1 polyadenylation signals, only the signal directing cleavage of PAS3 is conserved in the rat, again indicating that PAS3 is the constitutive polyadenylation site whereas PAS1, PAS2 and PAS4 can be used alternatively. This is further supported by the fact that the $7 \mathrm{~kb}$ transcript, which derives from transcripts using PAS3, is more strongly expressed than the $4.5 \mathrm{~kb}$ and $3.5 \mathrm{~kb}$ transcripts. Moreover, PAS3 is represented by multiple ESTs in the database that are derived from a variety of fetal and adult tissues (see additional file 1, Fig. 1a). No ESTs were found representing transcripts using PAS2 and PAS4 and only a few ESTs are present to indicate usage of PAS1. 
ESTs for PAS1 are mainly derived from stomach, suggesting tissue-specific usage of PAS1.

However, a definitive statement about relative expression levels of the alternatively polyadenylated MID1 transcripts cannot be made at this point which is due to the following reasons: First comparison of differently sized transcripts that are detected through northern blot analyses is limited because the signal intensity is influenced by the sizes of the respective transcripts. Second the sequences of the alternatively polyadenylated MID1 transcripts are partially overlapping, and thus cannot be amplified individually by RT-PCR experiments.

Interestingly, we show that promoter usage is linked to poly(A) site selection in the MID1 gene (Fig. 3a-f). This phenomenon cannot be explained solely by expression of tissue-specific polyadenylation factors although the relative levels of expression of polyadenylation factors and transcription factors might influence the poly(A) site selection in a given cell-type [17]. Splicing factors that have a role in 3'end formation, as suggested recently [1923] could contribute here. Also, chromatin-remodelling enzymes that can have both positive and negative roles in promoter regulation, elongation and termination could be involved [24]. In line with that hypothesis it has been suggested that predefined chromatin transcription units exist in yeast before transcription commences [24]. Furthermore, specific transcription factors that bind to both the promoter and poly(A) signal could play a role, which is supported by the observation that an increasing number of factors are essential for transcription and transcript termination $[24,25]$. It seems possible that all these mechanisms act together and build up a complex regulatory network that controls poly(A) site selection in order to ensure a tight control of gene expression. In the future the well characterized MID1 transcripts will be a suitable model for further investigation of this plausible hypothesis.

5'UTRs and 3'UTRs are implicated in the regulation of many aspects of mRNA function. 5'UTRs may contain upstream open reading frames which inhibit translation by restricting the access of ribosomes to the correct start codon [14]. Several upstream AUG codons are present in the different MID1 5'UTR exons and hence, it was suggested that differentially transcribed MID1 isoforms are translated at different levels [4]. Moreover, both 5'UTRs and 3'UTRs can contain specific sites to which regulatory RNAs or proteins bind. The composition of these sites ranges from short primary sequence elements to specific secondary structures $[14,26]$. Sequence analyses of the MID1 3'UTR revealed the existence of several cytoplasmic polyadenylation elements (CPEs). Cytoplasmic polyadenylation is a key mechanism affecting genes that are involved in synaptic plasticity and controlling mRNA translation during early development [10]. It is regulated by two cis-acting sequences, the CPE and the upstream element AAUAAA. Although it has been suggested that CPEs are usually located within 20-30 nucleotides upstream of the AAUAAA element, examples of mRNAs with much longer CPE-to-AAUAAA distances have been described, e.g. the CPE of $\mathrm{C} 11$, which resides 286 nucleotides upstream of the hexamer [27]. Of note, four of the six CPEs found in the MID1 3'UTR are conserved in other species (see additional file 4). Besides CPEs, the MID1 3'UTR contains multiple AU-rich elements (AREs) of the sequence ATTTA, several of which are conserved in other species (Fig. 4a). Like functionally relevant AREs of other genes [26], four of the conserved pentamers of the MID1 3 'UTR are embedded in much longer AU-rich sequences (Fig. 4a). AREs are well described sequence elements to which a range of different proteins can bind, e.g. AUF1, HuR and KSRP $[26,28]$. These proteins can influence stability and/or translation of the respective mRNAs. In a UV-crosslink assay we could identify several proteins that bind to the ARE1 motif of the human MID1 3'UTR. As the sizes of the identified proteins fit quite well with the sizes of several known ARE-binding proteins, such as HuR and AUF1, they were good candidates for regulating MID1 expression. In an RNA-protein pulldown assay we could indeed confirm binding of these proteins to the ARE1 motif of the MID1 3'UTR.

\section{Conclusion}

We found that mature mRNAs of the MID1 gene end at four different polyadenylation sites. The different 3'UTRs of the MID1 gene contain several evolutionary conserved sequence motifs, which suggests a contribution of the 3'UTRs to the mRNA stability and translation of the gene. In addition, we found that expression of the MID1 gene is differentially regulated by the concerted action of alternative promoters and alternative polyadenylation signals both during embryonic development and in the adult.

\section{Methods}

\section{RT-PCR, 3' and 5'-RACE, Northern Blot Analysis}

Total RNA from human testis was purchased from BioCat (BioCat GmbH, Heidelberg, Germany). Total RNA from fetal brain was purchased from Clontech. Total RNA from rat brain was kindly provided by Dr. Diego Walther. cDNA synthesis was performed as described previously [3]. $3 \mu \mathrm{l}$ from a total of $25 \mu \mathrm{l} \mathrm{cDNA}$ was used for PCR with primers annealing to different parts of the 3 '- and 5'UTRs of the MID1 gene (for primer sequences see additional file 6). First and nested PCRs were performed following the instructions of the Expand Long Template PCR System (Roche, Germany). PCR products were excised from the gel, purified using a Gel Extraction Kit (Qiagen, Germany), cloned into the pGEM-T Easy vector (Promega) 
and sequenced. 3 ' and 5'-Race experiments were performed as described previously [3]. Amplification of cDNA was carried out using primers that annealed to different parts of the human and rat MID1 3'UTRs. Primer sequences for RT-PCR, $3^{\prime}$ and 5'-Race experiments are given in additional files 5 and 6 .

Multiple-tissue Northern blots (Clontech) were hybridized with ${ }^{32}$ P-labeled DNA probes or riboprobes. Riboprobe nbPAS4 was synthesized by in vitro transcribing a PCR template corresponding to a sequence $5^{\prime}$ of PAS4. Primer sequences are given in additional file 7 . Hybridizations were carried out as described previously [3].

\section{In vitro transcription}

32P-labelled cRNAs or biotin-labelled cRNAs corresponding to the sense and antisense 70-307 3'UTR human MID1 were produced using purified PCR-amplified cDNA which included the T7 Polymerase promoter sequence and T7 polymerase (Promega) according to the manufacturer's procedure. Primer sequences are given in additional file 7 . In vitro transcribed probes were DNAse treated and Ethanol precipitated.

\section{UV crosslinking assay}

HeLa cells were lysed with ultrasound and centrifuged at $12,000 \times$ g 15 min. $4^{\circ} \mathrm{C}$. Reaction mixtures containing 20 $\mu \mathrm{g}$ of protein lysate in reaction buffer (5.2 mM HEPES [pH 7.9], $50 \mathrm{mM} \mathrm{KCl}, 10 \mathrm{mM}$ DTT, $5 \mathrm{mg} / \mathrm{ml}$ heparin, $1 \%$ glycerol, $40 \mu \mathrm{g} / \mathrm{ml}$ yeast tRNA) and $250.000 \mathrm{cpm}$ of radiolabeled probe were incubated for $10 \mathrm{~min}$. at room temperature, UV crosslinked for $10 \mathrm{~min}$. in a UV Stratalinker 1800 (Stratagene) and digested with $1 \mathrm{U}$ each of RNAse A and RNAse T1 for $15 \mathrm{~min}$. at $37^{\circ} \mathrm{C}$. Complexes were resolved by electrophoresis through SDS-10\% acrylamide gels, after denaturation at $95^{\circ} \mathrm{C}$ for $5 \mathrm{~min}$. Gels were dried and exposed to X-ray film.

\section{RNA-protein pulldown}

HeLa cells were lysed with ultrasound and centrifuged at $12,000 \times \mathrm{g} 15 \mathrm{~min} .4^{\circ} \mathrm{C}$. Reaction mixtures containing $200 \mu \mathrm{g}$ of protein lysate in TKM buffer $(20 \mathrm{mM}$ Tris [pH 7.5], $150 \mathrm{mM} \mathrm{KCl}, 5 \mathrm{mM} \mathrm{MgCl}_{2}$ ) supplemented with $1 \%$ NP40, $1 \mathrm{mM}$ DTT, complete protease inhibitor cocktail (Roche), $100 \mathrm{U}$ of RNasin (Promega) and $3 \mu \mathrm{g}$ of biotinlabelled probe were incubated for 1 hour at $4{ }^{\circ} \mathrm{C}$, followed by the addition of streptavidin magnetic beads and incubation for 2 hours at $4^{\circ} \mathrm{C}$. After washing and denaturation at $95^{\circ} \mathrm{C}$ for $5 \mathrm{~min}$. proteins were resolved by electrophoresis through SDS-10\% acrylamide gels. Gels were blotted on PVDF membranes and Westernblot analyses performed with antibodies directed against HuR (Santa Cruz) and AUF1 (Upstate).

\section{Bioinformatic analyses}

We used the UCSC Genome Browser March 2006 assembly to analyse the complex structure of the 3'UTR MID1 including repeat occurrence and evolutionary sequence conservation [29]. The longest 3'UTR, which spans all the shorter transcript variants, was scanned for potential polyadenylation signals [15] and known binding motifs for RNA-binding proteins (RBP) using BioPerl [30]. For detecting polyadenylation signals we used the upstream core element AATAAA and the downstream GU or U-rich element. For detecting of CPEs and AREs we used the minimal elements TTTTAT and ATTTA which have been shown to suffice for binding of interacting proteins $[26,10]$.

\section{Authors' contributions}

JW supervised the work and performed the 3' and 5'RACE, Northern Blot experiments, the UV-Assay and the RNA-protein pulldown. MK performed the RT-PCR experiments. SR and SK performed the bioinformatic analyses. RS and SS supervised the work. All authors read and approved the final manuscript.

\section{Additional material}

\section{Additional file 1}

Human ESTs indicate usage of PAS1 or PAS3. The table lists all ESTs for PAS1 and PAS3.

Click here for file

[http://www.biomedcentral.com/content/supplementary/14712199-8-105-S1.doc]

\section{Additional file 2}

Poly (A) signals that are in close proximity to the alternative poly (A) sites of the human and rat MID1 3'UTRs. This figure shows the composition of poly (A) signals for human and rat alternative poly (A) sites.

Click here for file

[http://www.biomedcentral.com/content/supplementary/14712199-8-105-S2.ppt]

\section{Additional file 3}

Conservation of the hexamers AAUAAA located upstream of the alternative MID1 polyadenylation sites in different species. Shown is an alignment of the hexamers located upstream of the alternative MID1 polyadenylation sites for different mammalian and other vertebrate species.

Click here for file

[http://www.biomedcentral.com/content/supplementary/14712199-8-105-S3.ppt]

\section{Additional file 4}

Conservation of cytoplasmic polyadenylation elements in different species. Shown is an alignment of the cytoplasmic polyadenylation elements located in the MID1 3 'UTR for different mammalian and other vertebrate species.

Click here for file

[http://www.biomedcentral.com/content/supplementary/14712199-8-105-S4.ppt] 


\section{Additional file 5 \\ List of primers used for $3^{\prime}$ and 5 'RACE. This table provides the sequences of primers used for $3^{\prime}$ and 5'RACE experiments. \\ Click here for file \\ [http://www.biomedcentral.com/content/supplementary/1471- 2199-8-105-S5.doc] \\ Additional file 6 \\ List of primers used for RT-PCR. This table provides the sequences of prim- ers used for RT-PCR experiments. \\ Click here for file \\ [http://www.biomedcentral.com/content/supplementary/1471- \\ 2199-8-105-S6.doc] \\ Additional file 7 \\ List of primers used for generating probes for Northern blot analysis and the UV-crosslink/RNA-protein pulldown assay. This table provides the sequences of primers used for generating probes for Northern blot analysis and $U V$-crosslink/RNA-protein pulldown assays \\ Click here for file \\ [http://www.biomedcentral.com/content/supplementary/1471- 2199-8-105-S7.doc]}

\section{Acknowledgements}

We thank Dr. Sybille Krauss and Dr. Daryl Henderson for critical reading of the manuscript, Dr. Diego Walther for kindly providing RNA from rat brain and Dr. Sigmar Stricker for helpful advice. The project was financed by the DFG (Deutsche Forschungsgemeinschaft, SFB 577, project C04; Project SCHW 829/4-I) and the Volkswagen-Stiftung. R.S. was supported by the Special Research Program SFB02 I-"Cell Proliferation and Cell Death in Tumors" of the Austrian Science Fund (FWF).

\section{References}

I. Schweiger S, Foerster J, Lehmann T, Suckow V, Muller YA, Walter G, Davies T, Porter H, van Bokhoven H, Lunt PW, Traub P, Ropers HH: The Opitz syndrome gene product, MIDI, associates with microtubules. Proc Natl Acad Sci U S A 1999, 96(6):2794-2799.

2. Trockenbacher A, Suckow V, Foerster J, Winter J, Krauss S, Ropers $\mathrm{HH}$, Schneider R, Schweiger S: MIDI, mutated in Opitz syndrome, encodes an ubiquitin ligase that targets phosphatase 2A for degradation. Nat Genet 200I, 29(3):287-294.

3. Winter J, Lehmann T, Krauss S, Trockenbacher A, Kijas Z, Foerster J, Suckow V, Yaspo ML, Kulozik A, Kalscheuer V, Schneider R, Schweiger S: Regulation of the MIDI protein function is finetuned by a complex pattern of alternative splicing. Hum Genet 2004, I | 4(6):54 I-552.

4. Landry JR, Mager DL: Widely spaced alternative promoters, conserved between human and rodent, control expression of the Opitz syndrome gene MID I. Genomics 2002, 80(5):499-508.

5. Dal Zotto L, Quaderi NA, Elliott R, Lingerfelter PA, Carrel L, Valsecchi V, Montini E, Yen CH, Chapman V, Kalcheva I, Arrigo G, Zuffardi O, Thomas S, Willard HF, Ballabio A, Disteche CM, Rugarli El: The mouse Mid I gene: implications for the pathogenesis of Opitz syndrome and the evolution of the mammalian pseudoautosomal region. Hum Mol Genet 1998, 7(3):489-499.

6. Quaderi NA, Schweiger S, Gaudenz K, Franco B, Rugarli El, Berger W, Feldman GJ, Volta M, Andolfi G, Gilgenkrantz S, Marion RW, Hennekam RC, Opitz JM, Muenke M, Ropers HH, Ballabio A: Opitz G/ BBB syndrome, a defect of midline development, is due to mutations in a new RING finger gene on Xp22. Nat Genet 1997, I 7(3):285-29I.

7. Richman JM, Fu KK, Cox LL, Sibbons JP, Cox TC: Isolation and characterisation of the chick orthologue of the Opitz syndrome gene, MidI, supports a conserved role in vertebrate development. Int J Dev Biol 2002, 46(4):44I-448.
8. Pinson L, Auge J, Audollent S, Mattei G, Etchevers H, Gigarel N, Razavi F, Lacombe D, Odent S, Le Merrer M, Amiel J, Munnich A, Meroni G, Lyonnet S, Vekemans M, Attie-Bitach T: Embryonic expression of the human MIDI gene and its mutations in Opitz syndrome. J Med Genet 2004, 4 I (5):38I-386.

9. Winter J, Lehmann T, Suckow V, Kijas Z, Kulozik A, Kalscheuer V, Hamel B, Devriendt K, Opitz J, Lenzner S, Ropers HH, Schweiger S: Duplication of the MIDI first exon in a patient with Opitz G/ BBB syndrome. Hum Genet 2003, I I 2(3):249-254.

10. Mendez R, Richter JD: Translational control by CPEB: a means to the end. Nat Rev Mol Cell Biol 200I, 2(7):52l-529.

II. Lopez de Silanes I, Zhan M, Lal A, Yang X, Gorospe M: Identification of a target RNA motif for RNA-binding protein HuR. Proc Natl Acad Sci U S A 2004, I 0 I (9):2987-2992.

12. Mitchell $P$, Tollervey D: mRNA stability in eukaryotes. Curr Opin Genet Dev 2000, I0(2): 193-198.

13. Tollervey D: Molecular biology: RNA lost in translation. Nature 2006, 440(7083):425-426.

14. Hughes TA: Regulation of gene expression by alternative untranslated regions. Trends Genet 2006, 22(3): I |9-122.

15. Carninci P, Sandelin A, Lenhard B, Katayama S, Shimokawa K, Ponjavic J, Semple CA, Taylor MS, Engstrom PG, Frith MC, Forrest AR, Alkema WB, Tan SL, Plessy C, Kodzius R, Ravasi T, Kasukawa T, Fukuda S, Kanamori-Katayama M, Kitazume Y, Kawaji H, Kai C, Nakamura M, Konno H, Nakano K, Mottagui-Tabar S, Arner P, Chesi A, Gustincich S, Persichetti F, Suzuki H, Grimmond SM, Wells CA, Orlando V, Wahlestedt C, Liu ET, Harbers M, Kawai J, Bajic VB, Hume DA, Hayashizaki $Y$ : Genome-wide analysis of mammalian promoter architecture and evolution. Nat Genet 2006, 38(6):626-635.

16. Ara T, Lopez F, Ritchie W, Benech P, Gautheret D: Conservation of alternative polyadenylation patterns in mammalian genes. BMC Genomics 2006, 7:189.

I7. Prasanth KV, Prasanth SG, Xuan Z, Hearn S, Freier SM, Bennett CF, Zhang MQ, Spector DL: Regulating gene expression through RNA nuclear retention. Cell 2005, I 23(2):249-263.

18. Tian B, Hu J, Zhang H, Lutz CS: A large-scale analysis of mRNA polyadenylation of human and mouse genes. Nucleic Acids Res 2005, 33(I):20I-2I 2.

19. Danckwardt S, Kaufmann I, Gentzel M, Foerstner KU, Gantzert AS, Gehring NH, Neu-Yilik G, Bork P, Keller W, Wilm M, Hentze MW, Kulozik AE: Splicing factors stimulate polyadenylation via USEs at non-canonical 3' end formation signals. Embo J 2007, 26(I I):2658-2669.

20. McCracken S, Lambermon M, Blencowe BJ: SRm I 60 splicing coactivator promotes transcript 3'-end cleavage. Mol Cell Biol 2002, 22(I): | $48-160$.

21. Evans D, Perez I, MacMorris M, Leake D, Wilusz CJ, Blumenthal T: A complex containing CstF-64 and the SL2 snRNP connects mRNA 3' end formation and trans-splicing in C. elegans operons. Genes Dev 200I, I 5(I 9):2562-257I.

22. Edmonds $M$ : $A$ history of poly $A$ sequences: from formation to factors to function. Prog Nucleic Acid Res Mol Biol 2002, 7 I :285-389.

23. Millevoi S, Loulergue C, Dettwiler S, Karaa SZ, Keller W, Antoniou $M$, Vagner S: An interaction between U2AF 65 and CF I(m) links the splicing and $3^{\prime}$ end processing machineries. Embo J 2006, 25(20):4854-4864.

24. Proudfoot $\mathrm{N}$ : New perspectives on connecting messenger RNA 3' end formation to transcription. Curr Opin Cell Biol 2004, I 6(3):272-278.

25. Maniatis T, Reed R: An extensive network of coupling among gene expression machines. Nature 2002, 4 I 6(6880):499-506.

26. Zhang T, Kruys V, Huez G, Gueydan C: AU-rich element-mediated translational control: complexity and multiple activities of trans-activating factors. Biochem Soc Trans 2002, 30(Pt 6):952-958.

27. Simon R, Richter JD: Further analysis of cytoplasmic polyadenylation in Xenopus embryos and identification of embryonic cytoplasmic polyadenylation element-binding proteins. Mol Cell Biol 1994, I 4( 1 2):7867-7875.

28. Brennan CM, Steitz JA: HuR and mRNA stability. Cell Mol Life Sci 200I, 58(2):266-277.

29. Kuhn RM, Karolchik D, Zweig AS, Trumbower H, Thomas DJ, Thakkapallayil A, Sugnet CW, Stanke M, Smith KE, Siepel A, Rosenbloom KR, Rhead B, Raney BJ, Pohl A, Pedersen JS, Hsu F, Hinrichs AS, Harte RA, Diekhans M, Clawson H, Bejerano G, Barber GP, Baertsch R, Haussler D, Kent WJ: The UCSC genome browser 
database: update 2007. Nucleic Acids Res 2007, 35(Database issue): $\mathrm{D} 668-73$.

30. Stajich JE, Block D, Boulez K, Brenner SE, Chervitz SA, Dagdigian C, Fuellen G, Gilbert JG, Korf I, Lapp H, Lehvaslaiho H, Matsalla C, Mungall CJ, Osborne BI, Pocock MR, Schattner P, Senger M, Stein LD, Stupka E, Wilkinson MD, Birney E: The Bioperl toolkit: Perl modules for the life sciences. Genome Res 2002, I2(10):16 II-1618.

Publish with Bio Med Central and every scientist can read your work free of charge

"BioMed Central will be the most significant development for disseminating the results of biomedical research in our lifetime. " Sir Paul Nurse, Cancer Research UK

Your research papers will be:

- available free of charge to the entire biomedical community

- peer reviewed and published immediately upon acceptance

- cited in PubMed and archived on PubMed Central

- yours - you keep the copyright

Submit your manuscript here:

http://www.biomedcentral.com/info/publishing_adv.asp
BiolMedcentral 